\title{
Emission of the terahertz electromagnetic wave from coherent longitudinal optical phonons in a GaAs buffer layer optically masked by a GaSb top epitaxial layer
}

\section{Hideo Takeuchi, Syuichi Tsuruta, Masaaki Nakayama}

\begin{tabular}{|c|l|}
\hline Citation & Applied Physics Letters, 98(15): 151905 \\
\hline Issue Date & 2011-04-13 \\
\hline Type & Journal Article \\
\hline Textversion & publisher \\
\hline Right & $\begin{array}{l}\text { C 2011 American Institute of Physics. This article may be downloaded for personal use } \\
\text { only. Any other use requires prior permission of the author and AIP Publishing. } \\
\text { The following article appeared in Applied Physics Letters and maybe found at } \\
\text { https://doi.org/10.1063/1.3574541 }\end{array}$ \\
\hline DOI & $10.1063 / 1.3574541$ \\
\hline
\end{tabular}

\section{Self-Archiving by Author(s)}

Placed on: Osaka City University Repository 


\title{
Emission of the terahertz electromagnetic wave from coherent longitudinal optical phonons in a GaAs buffer layer optically masked by a GaSb top epitaxial layer
}

\author{
Hideo Takeuchi, ${ }^{1, a)}$ Syuichi Tsuruta, ${ }^{2}$ and Masaaki Nakayama ${ }^{2}$ \\ ${ }^{1}$ Department of Electronic Systems Engineering, School of Engineering, The University of Shiga Prefecture, \\ 2500 Hassaka-cho, Hikone, Shiga 522-8533, Japan \\ ${ }^{2}$ Department of Applied Physics, Graduate School of Engineering, Osaka City University, \\ 3-3-138 Sugimoto, Sumiyoshi-ku, Osaka 558-8585, Japan
}

(Received 1 March 2011; accepted 16 March 2011; published online 13 April 2011)

We demonstrate that, in a $\mathrm{GaSb} / \mathrm{GaAs}$ epitaxial structure, the coherent longitudinal optical (LO) phonon in the GaAs layer optically masked by the GaSb top layer is observed utilizing terahertzelectromagnetic-wave spectroscopy. It is confirmed from a Raman scattering measurement that only the optical phonon in the GaSb layer is optically observable, where the photon energy of the excitation laser beam was almost the same as that of the femtosecond pulse pump beam for the terahertz wave measurement. In the terahertz wave measurement, the Fourier power spectrum of the terahertz waveform exhibits both the GaAs and the GaSb LO phonons; namely, the coherent LO phonon in the optically masked GaAs buffer layer is observed in the terahertz wave measurement. This fact demonstrates that the instantaneous surface potential modulation originating from the impulsive carrier excitation by the pump pulses reaches the GaAs buffer layer. Consequently, the above-mentioned surface potential modulation generates the coherent GaAs LO phonon. (C) 2011 American Institute of Physics. [doi:10.1063/1.3574541]

Terahertz-electromagnetic-wave spectroscopy gives an interesting issue on the detection and generation of coherent phonons. In the conventional Raman scattering process, phonons are generated directly by the illumination of the excitation laser beam. In the generation process of coherent longitudinal optical (LO) phonons, those are induced by the surge current of photogenerated carriers that changes a potential structure in equilibrium. ${ }^{1}$ The generation mechanism of the coherent phonons indicates that the substantial trigger for driving the coherent $\mathrm{LO}$ phonons is not the illumination of the pump beam but the resultant potential modulation. Accordingly, even in the case where a given layer is optically masked by an upper layer, it is considered that the coherent LO phonons can be generated through an instantaneous change in the potential. In the research field of the coherent LO phonons, the typical detection method is a reflection-type pump-probe technique. In this method, the coherent LO phonons are detected through its modulation effect on the reflectivity of the probe beam. It should be noted that, in usual, the pump and probe beams have the same photon energy. Accordingly, within the above-mentioned detection scheme, it is impossible to detect the coherent LO phonons that exist in the region deeper than the penetration depth of the probe beam. Owing to the above-mentioned background, the coherent LO phonons in the optically masked layer have been out of the target, in spite of the presence of various reports on the coherent phonons. ${ }^{2-7}$ In contrast, terahertz wave spectroscopy has a potential to directly detect the signals of the coherent LO phonons as terahertz electromagnetic waves. We note that the terahertz waves from the coherent LO phonons have been investigated by various groups; ${ }^{8-11}$ however, the probing of those in the optically masked layer

${ }^{a)}$ Electronic mail: takeuchi.h@e.usp.ac.jp. has been neglected. The scenario described above provides us an important aspect of the generation mechanism of the terahertz waves from the coherent LO phonons, which will make advances in terahertz science and technology.

In the present work, we explore the feasibility of detecting the terahertz wave from the coherent LO phonon in the undoped GaAs buffer layer optically masked by the $\mathrm{GaSb}$ top layer in a $\mathrm{GaSb} / \mathrm{GaAs}$ epitaxial structure. We have succeeded in detecting the terahertz wave from the coherent LO phonon in the GaAs buffer layer in addition to the coherent GaSb LO phonon. We discuss the generation mechanism of the coherent phonon in terms of the instantaneous modulation of the surface potential bending. We also compare the present results with the results of a reflection-type pumpprobe measurement.

The present sample, the $\mathrm{GaSb} / \mathrm{GaAs}$ epitaxial structure, was grown on a (001)-oriented semi-insulating GaAs substrate by molecular beam epitaxy. The thicknesses of the $\mathrm{GaSb}$ top layer and GaAs buffer layer were $900 \mathrm{~nm}$ and 200 $\mathrm{nm}$, respectively. The both layers were undoped. Consequently, the free carrier absorption of the terahertz wave is negligible in the GaSb layer. This fact means that the GaSb layer is transparent to the terahertz wave.

In advance to the terahertz wave measurement, we performed the Raman scattering measurement of the $\mathrm{GaSb} /$ GaAs structure in the back scattering configuration at room temperature in order to confirm that the GaAs layer is optically masked by the GaSb layer. The photon energy and power of the excitation beam were $1.58 \mathrm{eV}$ and $19 \mathrm{~mW}$, respectively. Figure 1 shows the Raman scattering spectrum of the present sample. Two bands are observed at the frequencies of 6.8 and $7.1 \mathrm{THz}$. The frequencies of the $\mathrm{GaSb}$ transverse optical (TO) and LO phonons are $6.8 \mathrm{THz}$ and 7.0 $\mathrm{THz}$, respectively, ${ }^{12}$ so that the observed Raman bands are assigned to the GaSb TO and LO phonons in order of fre- 


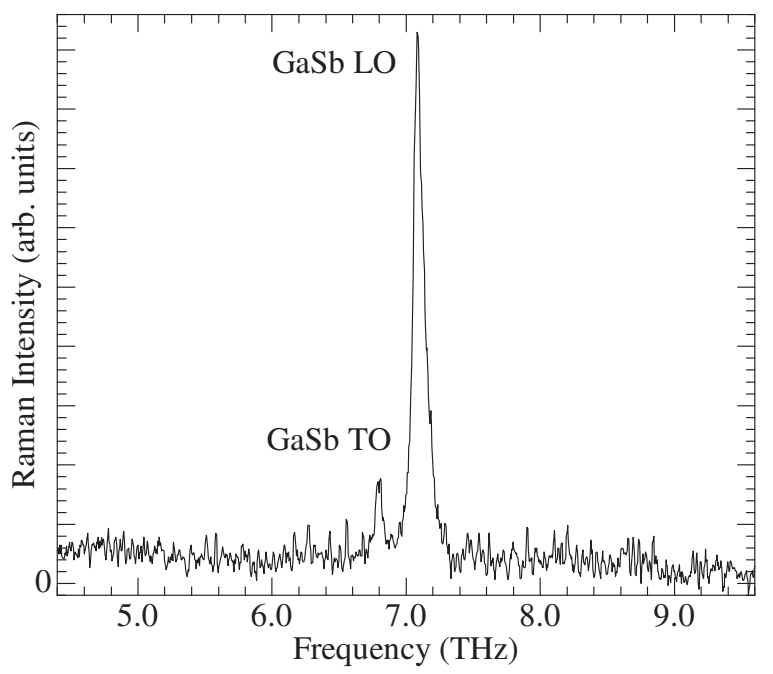

FIG. 1. Raman scattering spectrum of the $\mathrm{GaSb} / \mathrm{GaAs}$ structure measured at room temperature.

quency. The GaAs LO phonon with a frequency of $8.8 \mathrm{THz}$ (Ref. 13) is not observed in the Raman spectrum. The present result is reasonable because the penetration length of the excitation beam is $160 \mathrm{~nm}$ in the GaSb layer; ${ }^{14}$ namely, the GaSb top layer optically masks the GaAs buffer layer. We also note that, in principle, the TO-phonon band is forbidden in ideally (001)-oriented zincblende crystals. The observation of the GaSb TO-phonon band indicates the presence of an offset along the (001) direction in the GaSb layer. The responsible factor is attributed to the lattice mismatch between GaAs (lattice constant: $0.565 \mathrm{~nm}$ ) and GaSb $(0.610$ $\mathrm{nm}) .{ }^{12} \mathrm{It}$ is, therefore, considered that a certain amount of misorientation induced by the lattice mismatch exists in the $\mathrm{GaSb} / \mathrm{GaAs}$ interface. In fact, transmission-electronmicroscopy measurements of a GaSb/GaAs structure demonstrate that there are considerable dislocations in the interface region. ${ }^{15}$

The terahertz wave measurement was performed at room temperature. The humidity was kept at $\sim 10 \%$ during the measurement by purging with dry nitrogen gas. The pump beam was focused on the sample with the incidence angle of $45^{\circ}$. The diameter of the beam spot on the sample surface was $\sim 100 \mu \mathrm{m}$. The emitted terahertz wave was collected with use of two off-axis parabolic mirrors, and was detected by an optically gated dipole antenna with a gap of $6.0 \mu \mathrm{m}$ formed on a low-temperature-grown GaAs layer. The duration time of the laser pulses was about 50 fs. The powers of the pump and gate beams were fixed to $120 \mathrm{~mW}$ and $10 \mathrm{~mW}$, respectively. The repetition of the laser pulses was $90 \mathrm{MHz}$. The photon energies of both the beams were the same value of $1.57 \mathrm{eV}$. This value is almost the same as the excitation photon energy in the Raman scattering measurement. The scan range of the time delay was from -2.0 to $8.0 \mathrm{ps}$.

The terahertz waveform of the $\mathrm{GaSb} / \mathrm{GaAs}$ structure is shown in Fig. 2. Around the time delay of 0 ps, the monocycle oscillation pulse, the so-called first burst resulting from the surge current of photogenerated carriers, appears. The first burst is followed by the oscillatory profile. In order to highlight the oscillatory profile, the terahertz waveform in the range from 0.5 to $3.0 \mathrm{ps}$ is extracted and depicted in the inset of Fig. 2. It is evident that the oscillatory profile shows

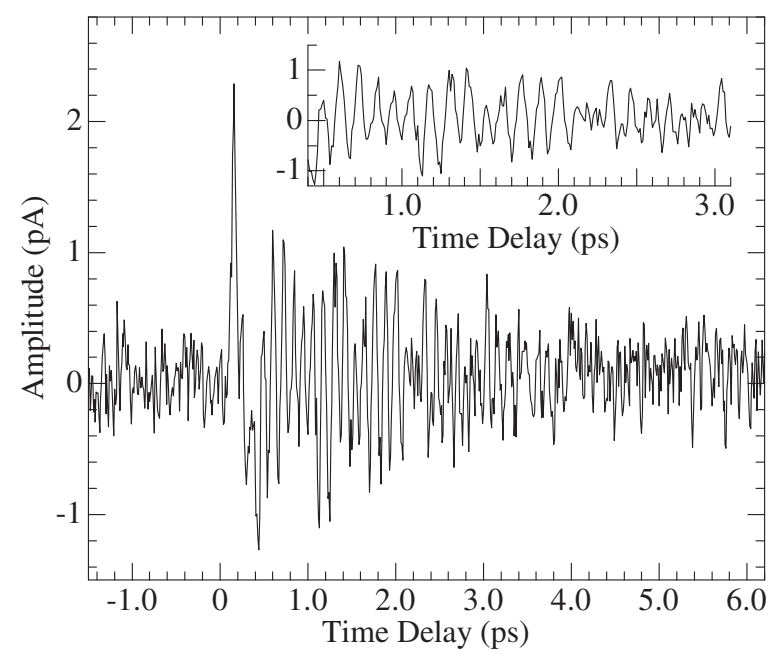

FIG. 2. Amplitude of the terahertz electromagnetic waveform of the GaSb/ GaAs structure as a function of time delay at room temperature. The inset shows the oscillatory profile between 0.5 and $3.0 \mathrm{ps}$ in order to highlight the beat pattern.

a beat. The appearance of the beat indicates that multiple oscillation modes are observed.

In order to clarify the observed modes, we performed the Fourier transform of the terahertz waveform. The Fourier power spectrum is shown in Fig. 3. The band of the first burst weakly appears around $2.0 \mathrm{THz}$. The band at $7.1 \mathrm{THz}$ is assigned to the coherent $\mathrm{GaSb} \mathrm{LO}$ phonon. It is emphasized that the GaAs LO phonon band emerges at $8.6 \mathrm{THz}$ in the Fourier power spectrum. Taking account of the fact that the frequency of the GaAs LO phonon in a single crystal is $8.8 \mathrm{THz}$, the shift in the phonon frequency $\Delta \omega$ is $-0.2 \mathrm{THz}$ in the present GaAs buffer layer. The value of $\Delta \omega$ corresponds to the tensile strain of $1.8 \times 10^{-2}$, assuming the biaxial strain induced by the lattice mismatch. In the estimation of the strain, the phonon deformation potential was taken from Ref. 16. We, therefore, conclude that the terahertz wave from the coherent GaAs LO phonons is observable even in the case where the GaAs buffer layer is optically masked by the $\mathrm{GaSb}$ layer.

Next, we compare the present result with the result of the reflection-type pump-probe measurement. According to Ref. 7, only the coherent GaSb LO phonon is observed in the

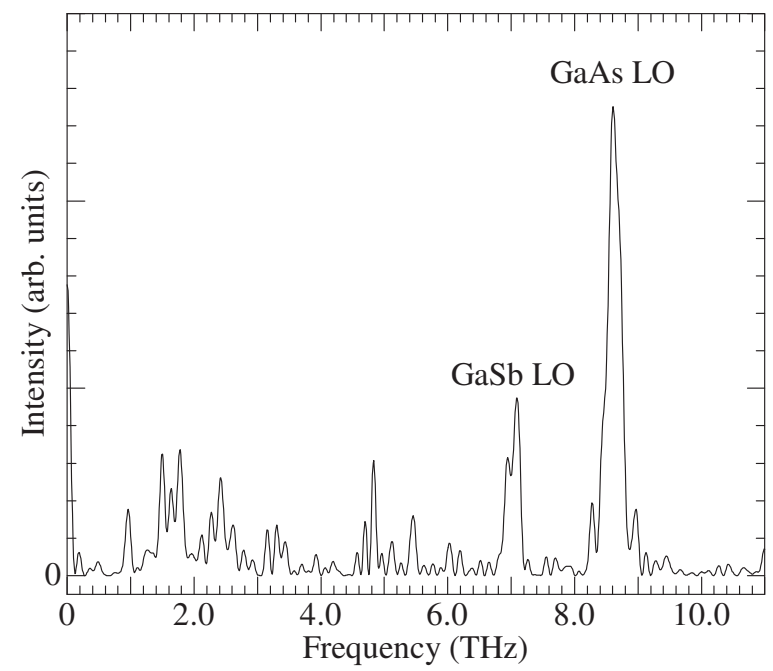

FIG. 3. Fourier power spectrum of the terahertz waveform shown in Fig. 2. 
reflection-type pump-probe measurement of a GaSb/GaAs structure using the pump-probe beams with a photon energy of $1.55 \mathrm{eV}$. The disappearance of the coherent GaAs LO phonon is attributed to the fact that, in the reflection-type pump-probe measurement, the probe beam cannot reach at the GaAs/GaSb interface. Thus, it is concluded that the terahertz wave measurement of the coherent LO phonon is applicable to probe the layers optically masked by the upper layer.

Finally, we discuss the generation mechanism of the coherent GaAs LO phonon. In general, compound semiconductors have a surface potential bending resulting from the surface Fermi level pinning. ${ }^{17}$ It is considered that, in an undoped semiconductor crystal, the region of the surface potential bending extends to several micrometers in the internal side of the crystal because carriers originating from unintentional dopants hardly screen the surface potential bending. The present $\mathrm{GaSb} / \mathrm{GaAs}$ structure has a total thickness of $1.1 \mu \mathrm{m}$; consequently, the surface potential bending reaches the GaAs buffer layer. The illumination of the pump beam, generates the dense carriers around the surface region, and, as a result, causes the instantaneous surface potential modulation across the $\mathrm{GaSb} / \mathrm{GaAs}$ interface. This phenomenon generates the coherent GaAs LO phonon.

In summary, we have investigated the Raman scattering spectrum and terahertz electromagnetic wave from the GaSb/ GaAs epitaxial structure. In the Raman scattering spectrum, the GaSb LO phonon band is observed, whereas the GaAs LO-phonon band is not detected. This is because the penetration length of the excitation beam is too short for the generation of the phonons in the GaAs layer. In contrast, the Fourier power spectrum of the terahertz wave exhibits both the coherent GaAs and GaSb LO phonons. The responsible factor for the generation of the coherent GaAs LO phonons has been attributed to the phenomenon that the instantaneous surface potential modulation originating from the impulsive carrier excitation by the pump pulse reaches the GaAs buffer layer. We have also compared the present result of the terahertz wave measurement with that of the conventional reflection-type pump-probe measurement in which only the coherent GaSb LO phonon is observable. From the abovementioned comparison, we conclude that the terahertz wave measurement of the coherent LO phonon is applicable to the investigation of the dynamical phenomena in the layers optically masked by the upper layer.

One of the authors, H.T., thanks the support of Grant-inAid for Young Scientists (B) under Grant No. 22760010 from the Japan Society for the Promotion of Science.

${ }^{1}$ For a review, T. Dekorsy, G. C. Cho, and H. Kurtz, in Light Scattering in Solids VIII, edited by M. Cardona and G. Güntherodt (Springer, Berlin, 2000), Chap. 4.

${ }^{2}$ G. C. Cho, W. Kütt, and H. Kurz, Phys. Rev. Lett. 65, 764 (1990).

${ }^{3}$ G. C. Cho, T. Dekorsy, H. J. Bakker, R. Hövel, and H. Kurz, Phys. Rev. Lett. 77, 4062 (1996).

${ }^{4}$ G. A. Garrett, T. F. Albrecht, J. F. Whitaker, and R. Marlin, Phys. Rev. Lett. 77, 3661 (1996).

${ }^{5}$ M. Hase, K. Mizoguchi, H. Harima, S. Nakashima, and K. Sakai, Phys. Rev. B 58, 5448 (1998).

${ }^{6}$ M. Nakajima, H. Harima, K. Morita, K. M. Ito, K. Mizoguchi, and E. E. Haller, Phys. Rev. B 63, 161304 (2001).

${ }^{7}$ H. Takeuchi, K. Mizoguchi, M. Nakayama, K. Kuroyanagi, T. Aida, M. Nakajima, and H. Harima, J. Phys. Soc. Jpn. 70, 2508 (2001).

${ }^{8}$ T. Dekorsy, H. Auer, C. Waschke, H. J. Bakker, H. G. Roskos, K. Kurtz, V. Wagner, and P. Grosse, Phys. Rev. Lett. 74, 738 (1995).

${ }^{9}$ M. Tani, R. Fukasawa, H. Abe, S. Matsuura, K. Sakai, and S. Nakashima, J. Appl. Phys. 83, 2473 (1998).

${ }^{10}$ M. Nakayama, S. Ito, K. Mizoguchi, S. Saito, and K. Sakai, Appl. Phys. Express 1, 012004 (2008).

${ }^{11}$ H. Takeuchi, J. Yanagisawa, S. Tsuruta, H. Yamada, M. Hata, and M. Nakayama, Jpn. J. Appl. Phys. 49, 082201 (2010).

${ }^{12}$ Semiconductors-Basic Data, edited by O. Madelung (Springer, Berlin, 1996).

${ }^{13} \mathrm{~N}$. Jusserand and M. Cardona, in Light Scattering in Solids V, edited by M. Cardona and G. Güntherodt (Springer, Berlin, 1989), Chap. 3.

${ }^{14}$ D. E. Aspnes and A. A. Studna, Phys. Rev. B 27, 985 (1983).

${ }^{15}$ Y. Wang, P. Ruterana, L. Desplanque, S. El Kazzi, and X. Wallart, J. Appl. Phys. 109, 023509 (2011).

${ }^{16}$ M. Nakayama, K. Kubota, T. Kanata, H Kato, S. Chika, and N. Sano, J. Appl. Phys. 58, 4342 (1985).

${ }^{17}$ H. H. Wieder, Surf. Sci. 132, 390 (1983). 